\title{
NÍVEIS DE RUÍDO NA LAVANDERIA DE UM HOSPITAL PÚBLICO
}

\section{Levels of noise in a public hospital laundry}

\author{
Monique Cantelli da Silva (1), Carolina Galoti Orlandi (2), Eliane Mi Chang (3), \\ Juliana Siviero ${ }^{(4)}$, Meliane Melina Pinto ${ }^{(5)}$, Patrícia Fernanda Staciarini Armellini ${ }^{(6)}$, \\ Thássia Silva dos Santos ${ }^{(7)}$, Vívian Baptista Luz ${ }^{(8)}$, Daniela Gil (9)
}

\begin{abstract}
RESUMO
Objetivo: mensurar os níveis de ruído em diferentes turnos da lavanderia do Hospital São Paulo (HSP) e prestar esclarecimentos sobre a saúde e a conservação auditivas aos funcionários e supervisores do setor. Método: trata-se de um estudo transversal descritivo. O ruído foi aferido por meio do medidor de pressão sonora na "Área Limpa" da lavanderia do HSP, em três períodos do dia: manhã (entre $7 \mathrm{~h}$ e $8 \mathrm{~h}$ ), tarde (entre 12h e 13h) e noite (entre 17h e 18h), por três dias e cada medição durou cinco minutos. Observou-se o uso de Equipamento de Proteção Individual (EPI) pelos funcionários em cada ambiente do hospital. Resultados: observou-se 11 máquinas (calandras, secadoras e lavadoras) no setor, que contribuiu para uma variação importante dos níveis de ruído na lavanderia (70 a $101 \mathrm{~dB}$ ) nos períodos aferidos. O uso de EPI pelos funcionários foi raro, tendo sido observada sua utilização por seis funcionários (12,76\%). Conclusão: independente do turno, os funcionários da lavanderia do Hospital São Paulo, os quais não fazem uso efetivo de EPIs, estão expostos diariamente a elevados níveis de pressão sonora, e que quanto maior o número de máquinas em funcionamento, maior foi o nível de ruído aferido.
\end{abstract}

DESCRITORES: Perda Auditiva Provocada por Ruído; Ruído Ocupacional; Ambiente de Instituições de Saúde; Ambiente de Trabalho

(1) Aluna do Curso de Graduação em FonoaudioFESP, São Paulo, SP.

(2) Aluna logia da Universidade Federal de São Paulo, UNI do Curso de Graduação em Fonoaudiologia da Universidade Federal de São Paulo, UNIFESP, São Paulo, SP.

(3) Aluna do Curso de Graduação em Fonoaudiologia da Universidade Federal de São Paulo, UNIFESP, São Paulo, SP.

(4) Aluna do Curso de Graduação em Fonoaudiologia da Universidade Federal de São Paulo, UNIFESP, São Paulo, SP.

(5) Aluna do Curso de Graduação em Fonoaudiologia da Universidade Federal de São Paulo, UNIFESP, São Paulo, SP.

(6) Aluna do Curso de Graduação em Fonoaudiologia da Universidade Federal de São Paulo, UNIFESP, São Paulo, SP.

(7) Aluna do Curso de Graduação em Fonoaudiologia da Universidade Federal de São Paulo, UNIFESP, São Paulo, SP.

(8) Aluna do Curso de Graduação em Fonoaudiologia da Universidade Federal de São Paulo, UNIFESP, São Paulo, SP.

(9) Fonoaudióloga; Professora Adjunta do Departamento de Fonoaudiologia da Universidade Federal de São Paulo, UNIFESP, São Paulo, SP; Doutora em Ciências pela Universidade Federal de São Paulo.

Conflito de interesses: inexistente

\section{INTRODUÇÃO}

As alterações auditivas devido à exposição ao ruído podem ser divididas em três tipos, a saber: o trauma acústico, mudanças temporárias e mudanças permanentes do limiar auditivo ${ }^{1}$.

O ruído pode ser dividido em contínuo ou intermitente, isto é, todo e qualquer ruído que não está classificado como ruído de impacto ou impulsivo. O ruído de impacto apresenta picos de energia acústica de duração inferior a um segundo, a intervalos superiores a um segundo. Entretanto, as normas sobre o assunto (NR 15 da Portaria n. 3.214 e norma da FUNDACENTRO) não diferenciam o ruído contínuo do intermitente para fins de avaliação quantitativa desse agente ${ }^{2,3,4}$.

Faz-se importante a quantificação do impacto dos efeitos deletérios do trabalho sobre a saúde dos trabalhadores para chamar a atenção da ocupação como causa de doença, subsequentemente o envolvimento necessário do setor de saúde que deve apreender o significado desse impacto, passando a ter maior participação interceptando, prevenindo, 
controlando e erradicando as causas de doenças ocupacionais ou que agravam a morbidade dos trabalhadores. Sendo assim, os níveis de ruído no ambiente hospitalar podem ser considerados potencialmente danosos ao ser humano ${ }^{5}$.

Além da perda auditiva, há relatos na literatura de que a exposição ao ruído intenso está associada a zumbido e tontura, assim como a várias manifestações sistêmicas, tais como elevação do nível geral de vigilância, aceleração da frequência cardíaca e respiratória, alteração da pressão arterial e da função intestinal, dilatação das pupilas, aumento do tônus muscular, aumento da produção de hormônios tireoidianos e estresse ${ }^{6,7,8}$.

Os acidentes do trabalho são o maior agravo à saúde dos trabalhadores. Constituem um importante problema de saúde pública, tanto em países em desenvolvimento, quanto em países desenvolvidos. Em estudo realizado foi encontrada associação positiva entre PAIR e acidentes ocupacionais ${ }^{9}$.

Em longos anos de prática clínica é possível constatar frequente exposição do trabalhador a condições adversas de trabalho, com lesões irreversíveis de órgãos sensoriais. De um lado nota-se grande resistência do trabalhador ao uso de protetores, tanto para as orelhas, quanto para os olhos, face, mãos, pés, etc. De outro lado, constata-se um grande descaso do empregador em oferecer uma proteção adequada aos empregados. $O$ médico do trabalho precisa esforçar-se para a melhoria do ambiente de trabalho e para que o trabalhador use proteção ${ }^{10}$.

Segundo a legislação trabalhista brasileira, a população mais suscetível a adquirir PAIR são trabalhadores expostos a elevados níveis de pressão sonora nas indústrias e empresas (acima de $85 \mathrm{~dB}$ NPS) ${ }^{8}$.

Já para ambientes hospitalares, os níveis aceitáveis de ruído variam de 35 a $45 \mathrm{~dB}(\mathrm{~A})$ segundo a norma da Associação Brasileira de Normas Técnicas (ABNT) NBR 10152/1987. Espera-se que os níveis de ruído em um hospital não muito movimentado devam encontrar-se entre 40 e $50 \mathrm{~dB}(\mathrm{~A})$; em um ambiente intermediário entre 50 a $60 \mathrm{~dB}(\mathrm{~A})$, e em um ambiente ruidoso, entre 60 a $70 \mathrm{~dB}(\mathrm{~A})^{3}$.

A PAIR saiu dos limites das grandes indústrias e está invadindo os hospitais. A não observância das leis regulamentares para o ruído industrial continua existindo; o que mudou foi o local e o público atingido pela PAIR. Estudo mostra que há prevalência de PAIR em funcionários de lavanderia de hospital universitário, reafirmando a importância da avaliação audiométrica de rotina como instrumento rápido, confiável e de baixo custo na prevenção e diagnóstico da PAIR ${ }^{11}$.
A realização de exames audiométricos periódicos permite o acompanhamento da saúde auditiva dos trabalhadores, identificando o desencadeamento ou agravamento de uma perda auditiva.

A velocidade da progressão da PAIR é também um fator que determina a eficácia das medidas de proteção adotadas e a necessidade da aplicação de outras medidas. Os efeitos extra-auditivos também devem ser considerados nesta avaliação.

Frente ao exposto, os objetivos deste estudo foram mensurar os níveis de ruído em diferentes turnos da lavanderia do Hospital São Paulo (HSP) e prestar esclarecimentos sobre a saúde e a conservação auditivas aos funcionários e supervisores do setor.

\section{MÉTODO}

Trata-se de um estudo transversal descritivo, realizado na lavanderia do Hospital São Paulo.

A lavanderia do HSP divide-se em "Área Limpa" e "Área Suja". A "Área Limpa", na qual foi realizada esta pesquisa, é constituída por quatro lavadoras, quatro secadoras, duas calandras (máquinas para passar roupas) e uma dobradeira (máquina para dobrar roupas).

Os funcionários são divididos em dois turnos de trabalho: $8 \mathrm{~h}$ às $18 \mathrm{~h}$ e das $18 \mathrm{~h}$ às $8 \mathrm{~h}$. Alguns poucos funcionários são diaristas e trabalham das $7 \mathrm{~h}$ às $16 \mathrm{~h}$.

Foram aferidos os níveis de ruído na "Área Limpa" em três dias da semana e em três períodos distintos no dia, às 8 horas (manhã), às 12 horas (tarde) e às 18 horas (noite).

Os critérios adotados para as aferições dos níveis do ruído foram:

- medições durante cinco minutos nesses períodos, nos corredores da lavanderia e entre as máquinas;

- registros dos níveis mínimos e máximos de pressão sonora encontrados;

- registro do número de máquinas em funcionamento e do tipo de funcionamento do maquinário (lavagem, centrifugação, secagem).

As pesquisadoras dividiram-se em duplas para aferir os níveis de ruído do local nos distintos dias, os quais foram agendados previamente com a supervisora da lavanderia.

As medições dos níveis de pressão sonora foram realizadas com decibelímetro modelo RadioShack Digital Sound-Level Meter, utilizado conforme especificação do fabricante.

O critério de avaliação foi realizado com circuito de compensação "A" e resposta lenta ("Slow"), conforme recomendação da norma regulamentadora NR 15 - anexo 1, da Portaria 3.214/78 3,4. 
Durante a aferição do ruído foram observados os seguintes aspectos do ambiente:

- número de funcionários por período;

- uso de equipamentos de proteção individual (EPIs);

- número de horas de trabalho;

- horário de maior intensidade sonora (picos de ruído).

Foi elaborada e realizada orientação sobre prevenção e conservação auditivas, para a qual foram utilizados panfletos (Figura 1) e esclarecimentos aos funcionários sobre o sistema auditivo, o ruído, sobre as consequências auditivas e não auditivas da exposição diária ao ruído intenso. Foram destacados:

- importância da audição;

- anatomia e fisiologia do ouvido;

- riscos da exposição ao ruído;

- avaliação audiológica;

- perda auditiva;

- programa de conservação auditiva;

- protetores auditivos.

A orientação foi realizada por meio da entrega de panfletos para cada trabalhador e apresentação de diferentes EPIs, com explicação de cada equipamento. A orientação foi realizada em dois dias para envolver o maior número de funcionários possível.

Essa pesquisa foi submetida ao Comitê de Ética em Pesquisa da Universidade Federal de São Paulo (protocolo n. ${ }^{\circ}$ 1975/08), e os participantes voluntários concordaram em assinar o Termo de Consentimento Livre e Esclarecido atendendo às Normas para a Realização de Pesquisa em Seres Humanos, Resolução 196/96, do Conselho Nacional de Saúde, de 10 de outubro de 1996.

Os dados obtidos nas medições realizadas durante os três períodos foram organizados em tabelas e dispostos em gráficos e comparados entre si, não fazendo uso de método estatístico por tratar-se de análises descritivas.

\section{RESULTADOS}

Os resultados apresentados (Tabelas 1 e 2 ) referem-se aos níveis mínimos e máximos de ruído aferidos nos três distintos turnos na lavanderia e o número de funcionários com EPI (Tabela 4).

Observa-se que os níveis de ruído encontrados variaram entre 70 e $101 \mathrm{~dB}$ NPS.

Durante os três dias de mensuração do nível de ruído na Área Limpa da lavanderia do HSP, foi observado um total de 47 funcionários, dos quais apenas três funcionários do período da tarde do $2^{\circ}$ dia e três funcionários também do período da tarde do $3^{\circ}$ dia estavam utilizando EPI, totalizando seis funcionários (12,76\%) (Tabela 4).

Durante as mensurações, três secadoras, duas calandras e três lavadoras estavam em funcionamento concomitante durante o dia, das dez máquinas existentes no local. Cabe colocar que, das máquinas da lavanderia, as que emitiram maior nível de ruído foram as lavadoras, no momento em que estavam centrifugando, cujo pico de ruído chegou a 94 dB NPS. Na Tabela 3 estão

Tabela 1 - Valores mínimos de ruído aferidos na lavanderia nos três diferentes turnos (dB NPS)

\begin{tabular}{cccc}
\hline & \multicolumn{3}{c}{ Mínimo Nível de Ruído (dB) } \\
\cline { 2 - 4 } & 10 dia & 20 dia & 3dia \\
\hline Manhã & 81 & 82 & 90 \\
Tarde & 82 & 82 & 76 \\
Noite & 70 & 80 & 80 \\
\hline
\end{tabular}

Legenda: $\mathrm{dB}$ = decibel; NPS = Nível de Pressão Sonora

Tabela 2 - Valores máximos de ruído aferidos na lavanderia nos três diferentes turnos (dB NPS)

\begin{tabular}{cccc}
\hline & \multicolumn{3}{c}{ Máximo Nível de Ruído (dB) } \\
\cline { 2 - 5 } & $\mathbf{1 0}$ dia & 20 dia & 30dia \\
\hline Manhã & 91 & 94 & 98 \\
Tarde & 94 & 97 & 90 \\
Noite & 96 & 99 & 101 \\
\hline
\end{tabular}

Legenda: $\mathrm{dB}=$ decibel; NPS = Nível de Pressão Sonora 
Tabela 3 - Classificação e quantificação das máquinas em funcionamento durante as medições nos três períodos

\begin{tabular}{cccccc}
\hline & \multicolumn{5}{c}{ Quantidade de Máquinas em Funcionamento } \\
\cline { 2 - 6 } & Secadora & Calandra & Lavadora & Dobradeira & Total \\
\hline 1ㅇ dia manhã & $3 / 4$ & $2 / 2$ & $3 / 4$ & 0 & 8 \\
1ㅇ dia tarde & $4 / 4$ & $2 / 2$ & $2 / 4$ & 0 & 8 \\
10 dia noite & $3 / 4$ & $2 / 2$ & $3 / 4$ & 0 & 8 \\
20 dia manhã & $4 / 4$ & $2 / 2$ & $2 / 4$ & 0 & 8 \\
2 dia tarde & $4 / 4$ & $2 / 2$ & $2 / 4$ & 0 & 8 \\
20 dia noite & $4 / 4$ & $2 / 2$ & $1 / 4$ & 0 & 7 \\
3o dia manhã & $4 / 4$ & $2 / 2$ & $3 / 4$ & 0 & 9 \\
3 dia tarde & $1 / 4$ & $2 / 2$ & $3 / 4$ & 0 & 6 \\
3 dia noite & $4 / 4$ & $2 / 2$ & $3 / 4$ & $1 / 1$ & 10 \\
\hline
\end{tabular}

Tabela 4 - Resultados do número de usuários de EPI nos três turnos

\begin{tabular}{cccc}
\hline & \multicolumn{3}{c}{ Uso de EPIs } \\
\cline { 2 - 4 } & $\mathbf{1 0}$ dia & $\mathbf{2}^{\circ}$ dia & 3dia \\
\hline Manhã & $0 / 3$ & $0 / 4$ & $0 / 6$ \\
Tarde & $0 / 4$ & $3 / 9$ & $3 / 9$ \\
Noite & $0 / 1$ & $0 / 3$ & $0 / 8$ \\
\hline
\end{tabular}

Legenda: EPI = Equipamento de Proteção Individual

dispostas a quantidade e os tipos de máquinas em funcionamento durante as medições.

A partir das aferições foi elaborado um folder para a conscientização do risco dos ruídos ocupacionais aos quais os trabalhadores estão expostos (Figura 1), os quais foram distribuídos aos funcionários e à supervisora do setor.

\section{DISCUSSÃO}

Um indivíduo pode adquirir uma perda auditiva induzida por ruído quando exposto a um nível de ruído igual ou superior a $85 \mathrm{~dB}(\mathrm{~A})$ diários como menciona a legislação brasileira (NR 15 - anexo 1, da Portaria 3.214/78) ${ }^{1,3,4}$.

Sabendo que o período de trabalho de cada funcionário da lavanderia do Hospital São Paulo é de no mínimo oito horas diárias, podemos inferir que se os mesmos estiverem expostos a níveis de ruído excessivos por um turno completo há uma grande probabilidade de adquirirem perda auditiva induzida por ruído (PAIR) e, consequentemente, dificuldades comunicativas decorrentes da perda auditiva neurossensorial, tais como: problemas na compreensão da fala, isolamento, constrangimento, diminuição da participação social e familiar, prejudicando a qualidade de vida e integração social ${ }^{12}$.

No primeiro dia em que foi realizada a aferição, observou-se que o período em que foi encontrado maior nível de pressão sonora foi o período da noite (96 dB) (Tabela 2). É importante ressaltar que os indivíduos que estavam trabalhando nesse período não utilizavam EPI.

Ainda no primeiro dia, de acordo com os resultados encontrados na Tabela 1, verificou-se que o valor mínimo do nível de pressão sonora também ocorreu no período da noite, sendo este igual a 70 $\mathrm{dB}$, o que, segundo a literatura e a legislação, não acarretaria risco de PAIR para os funcionários do período, se o nível de ruído fosse constante ${ }^{1,3,4}$.

Os níveis mínimos e os máximos de ruído aferidos (Tabelas 1 e 2), quase que em sua totalidade, foram encontrados no mesmo período (noite), demonstrando que neste período as campanhas de conservação auditiva devem ser intensificadas, apesar das realizadas pelo departamento de medicina do trabalho do hospital. Para estas campanhas, seria essencial a participação de um fonoaudiólogo para que os prejuízos decorrentes da exposição a ruído pudessem ser abordados de forma mais adequada do que o simples fornecimento de EPI. 


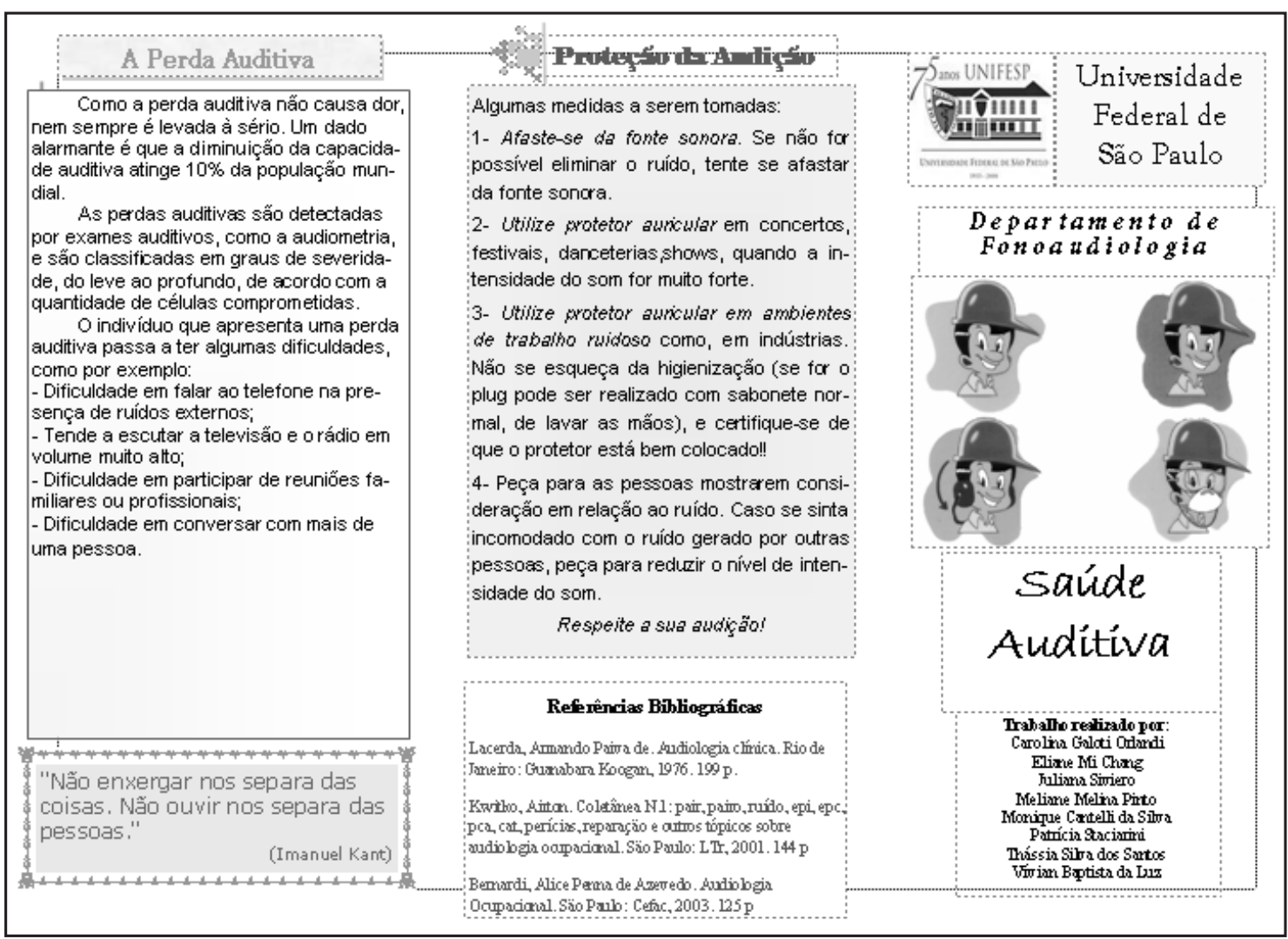

Figura 1 - Parte externa do folder

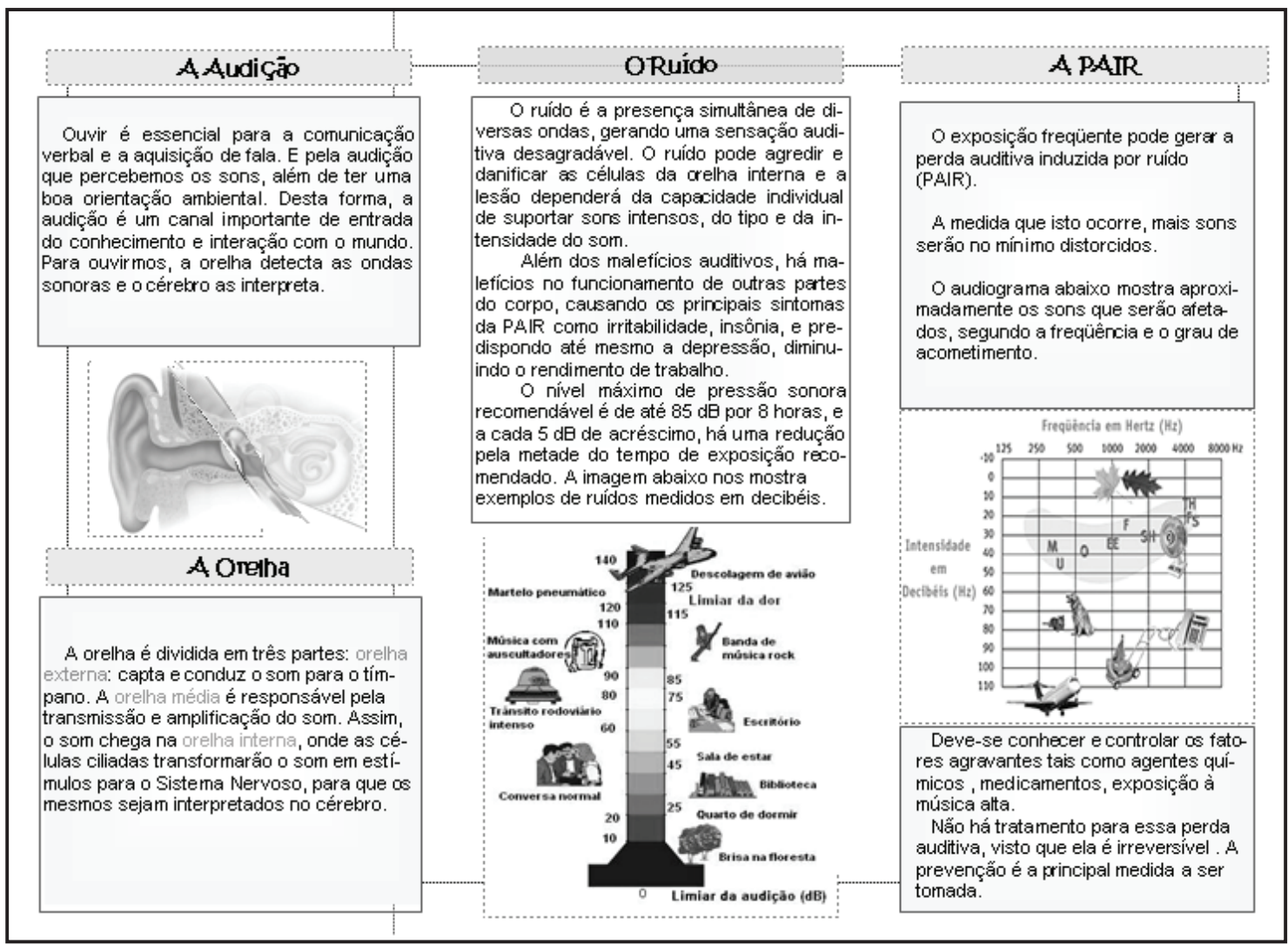


As oscilações nos níveis do ruído, aferidas em todos os turnos de trabalho permitiram caracterizar o ruído daquele ambiente como intermitente, o que pode colocar em risco a audição dos trabalhadores $^{2}$.

A Tabela 1 ainda demonstrou que os valores mínimos de ruído encontrados não foram prejudiciais ou alarmantes, no entanto estavam muito próximos do valor limite de $85 \mathrm{~dB}$. Há apenas uma exceção: o valor de $90 \mathrm{~dB}$ encontrado no $3^{\circ}$ dia de medição no período da manhã.

As Tabelas 1 e 2 revelaram a grande variação dos resultados encontrados (valores mínimos e máximos) que foram de risco para desenvolvimento de PAIR, indicando a necessidade de implementação de Programa de Conservação Auditiva (PCA) a fim de se evitar a instalação e a progressão de uma perda auditiva, a qual pode dificultar a recepção da linguagem oral ${ }^{5-11,13-15}$.

Pode-se concluir que o maior nível de ruído ocorreu no período da noite, ultrapassando, todos os dias, o valor de $85 \mathrm{~dB}$, que pode ser justificado pela maior quantidade de maquinário em funcionamento (Tabela 3).

Alguns autores destacaram a magnitude das estimativas de razão de taxa de incidência de acidentes do trabalho obtidas em trabalhadores expostos ao ruído em seu ambiente laboral ${ }^{14}$. No referido estudo, os trabalhadores que relataram às vezes estarem expostos ao ruído intenso, o risco relativo de acidentar-se foi 3,7 vezes $(1,8-7,4)$, enquanto que para aqueles que relataram sempre estarem expostos, o risco relativo foi estimado como 5,0 vezes maior $(2,8-8,7)$. O achado do referido estudo justifica o investimento em PCAs, do mesmo modo que as aferições realizadas neste trabalho, a fim de evitar danos e/ou perdas auditivas nos trabalhadores.

Como pode ser observado, os trabalhadores da lavanderia são de risco para desenvolver ou agravar uma PAIR, devido aos níveis elevados de pressão sonora encontrados nos distintos turnos de trabalho e o tempo de exposição contínuo, o que pode ser confirmado por meio de estudos com testes audiométricos ocupacionais com empregados de um hospital geral e comparação de grupos com e sem exposição em ambiente de trabalho hospitalar ${ }^{10,15}$.

Faz-se importante ressaltar o uso de EPI em todos os períodos, visto que, embora no período da tarde houvesse funcionários fazendo uso de tais equipamentos (Tabela 4), estes não correspondem à totalidade dos funcionários do período. Apesar dos funcionários receberem o equipamento de proteção individual auricular em sua admissão e ao longo do período de trabalho, os mesmos não recebem treinamento adequado, com estratégias educacionais e de motivação, para sua utilização, o que reflete em um uso pouco efetivo do EPI.

Os trabalhadores tiraram algumas dúvidas quanto ao material levado e abordado, aparentemente mostrando interesse aquém do esperado.

\section{CONCLUSÕES}

Com base nos resultados obtidos neste trabalho, foi possível concluir que, independente do turno (manhã, tarde e noite), os funcionários da lavanderia do Hospital São Paulo, os quais não fazem uso efetivo de EPIs, estão diariamente expostos a elevados níveis de pressão sonora, e que quanto maior o número de máquinas em funcionamento, maior é o nível de ruído.

\section{AGRADECIMENTOS}

À Prof. ${ }^{a}$ Dr. ${ }^{a}$ Renata Coelho Scharlach, pelo empréstimo do medidor de pressão sonora, sem o qual não poderíamos realizar esse trabalho, além de todo apoio dispensado.

À equipe da lavanderia do Hospital São Paulo, que gentilmente participou dessa pesquisa. 


\begin{abstract}
Purpose: to measure the noise levels in different shifts of Hospital São Paulo's (HSP) laundry and provide information on health and hearing conservation to the employees and supervisors of the sector. Method: this is a descriptive transversal study. The noise levels were checked using a Sound Pressure Meter in the "Clean Area" of the laundry, in three periods of the day: morning (between 7am and $8 \mathrm{am}$ ), afternoon (between 12pm and 1pm) and night (between $5 \mathrm{pm}$ and $6 \mathrm{pm}$ ), in three different days and each measurement lasted five minutes. The examiners also checked the use of Personal Protection Equipment (PPE) by the employees. Results: we observed 11 machines (skylarks, dryers and washers) in the sector, which contribute to an important variation of noise levels in the laundry (70 to $101 \mathrm{~dB}$ ) in the checked periods. The use of PPE by employees was rare, observing the use by only six employees (12.76\%). Conclusions: regardless of the shift, Hospital São Paulo laundry's employees, who do not effectively use PPEs, are daily exposed to high levels of sound pressure and as the number of machines in operation increased, the noise levels were also higher.
\end{abstract}

KEYWORDS: Hearing Loss, Noise-Induced; Noise, Occupational; Health Facility Environment; Working Environment

\section{REFERÊNCIAS}

1. Melnick W. Saúde auditiva do trabalhador. In: Katz J. Tratado de audiologia. 4.ed. São Paulo: Manole; 1999. p.529-47.

2. NHO 01. Série de normas de higiene ocupacional. Coordenação de Higiene do Trabalho da FUNDACENTRO - Projeto difusão de informações em higiene do trabalho. 1997/1998.

3. Equipe Atlas. Segurança e medicina do trabalho. 56. ed. São Paulo: Editora Atlas S.A.; 2005.

4. Brasil. Livro/Manual na internet: Saúde do Trabalhador - Protocolos de Complexidade Diferenciada 5. Série A. Normas e Manuais Técnicos. Ministério da Saúde - Secretaria de Atenção à Saúde. Departamento de Ações Programáticas Estratégicas. 2006. Disponível em: http://bvsms. saude.gov.br/bvs/publicacoes/06_0444_M.pdf

5. Otenio MH, Cremer E, Claro EMT. Intensidade de ruído em hospital de 222 leitos na 18 ${ }^{\mathrm{a}}$ Regional de Saúde - PR. Rev. Bras. Otorrinolaringol. 2007; 73(2):245-50.

doi: $10.1590 / S 1516-18462010005000050$

RECEBIDO EM: 18/11/2009

ACEITO EM: 05/03/2010

Endereço para correspondência:

Monique Cantelli da Silva

Rua Botucatu, 802

São Paulo - SP

CEP: 04023-062

E-mail: moniquecantelli@gmail.com
6. Dias A, Cordeiro R, Corrente JE, Gonçalves CGO. Associação entre perda auditiva induzida pelo ruído e zumbidos. Cad. Saúde Pública. 2006; 22(1):63-8. 7. Ogido R, Costa EA, Machado HC. Prevalência de sintomas auditivos e vestibulares em trabalhadores expostos a ruído ocupacional. Rev. Saúde Pública. 2009; 43(2):377-80.

8. Araújo SA. Perda auditiva induzida pelo ruído em trabalhadores de metalúrgica. Rev. Bras. Otorrinolaringol. 2002; 68(1):47-52.

9. Dias A, Cordeiro R, Gonçalves CGO. Exposição ocupacional ao ruído e acidentes do trabalho. Cad. Saúde Pública. 2006 Out; 22(10): 2125-2130.

10. Leme OLS. Estudo audiométrico comparativo entretrabalhadores deárea hospitalarexpostosenão expostos a ruído. Rev. Bras. Otorrinolaringol. 2001; 67(6):837-43.

11. Curado JAF, Rabelo WCSV, Alves W, Perini RF, Siqueira PH. A incidência de PAIR na lavanderia de um hospital universitário. Arq. Otorrinolaringol. 2001 Abr/Jun; 5(2): 113-6.

12. Amorim RMC, Almeida K. Estudo do benefício e da aclimatização em novos usuários de próteses auditivas. Pró-Fono. 2007; 19(1):39-48.

13. Marchiori LLM, Rego Filho EA, Matsuo T. Hipertensão como fator associado à perda auditiva. Rev. Bras. Otorrinolaringol. 2006; 72(4):533-40.

14. Cordeiro R, Clemente APG, Diniz CS, Dias A. Exposição ao ruído ocupacional como fator de risco para acidentes do trabalho. Rev. Saúde Pública. 2005; 39(3):461-6.

15. Kwitko A, Ferreira PG, França MT, Zanzini C, Steggiorin S. Perdas auditivas ocupacionais: análise de variáveis e diagnóstico. Rev. Bras. Med. 1996; 3:151-64. 